\title{
Modulation of the Nonlinear Optical Properties of Dibenzo[hi,st ]ovalene by Peripheral Substituents
}

\author{
Giuseppe M. Paternò ${ }^{1}$, Luca Nicoli ${ }^{2}$, Qiang Chen ${ }^{3}$, Klaus Müllen ${ }^{3}$, Akimitsu Narita ${ }^{3}$, Guglielmo Lanzani ${ }^{1,2}$ and \\ Francesco Scotognella ${ }^{1}$
}

${ }^{1}$ Istituto Italiano di Tecnologia, Center for Nano Science and Technology, Milano, 20133, Italy

${ }^{2}$ Politecnico di Milano, Department of Physics, Milano, 20133, Italy

${ }^{3}$ Max Planck Institute for Polymer Research, Mainz, 55128, Germany

\begin{abstract}
Dibenzo[hi,st] ovalene (DBOV) is a nanographene molecule with quasi-zero dimensional electronic confinement, which displays relatively high oscillator strength, remarkable photostability and optical gain property. For these reasons, DBOV has been proposed as gain medium and active material for achieving strong exciton-photon coupling in microcavity. Here, we study the stimulated emission properties of three DBOV derivatives with different substitution patterns. We found that these molecules likely undergo ultrafast intermolecular charge transfer processes occurring within their $\pi$ aggregates, which ultimately leads to quenching of stimulated emission and increase of the amplified spontaneous emission threshold. These effects can be minimized by installing bulky substituents on the peripheries that prevent $\pi-\pi$ stacking. We can thus selectively favor either the luminescence/gain properties or the charge transport features by engineering the side groups.
\end{abstract}

Keywords: nanographene; charge transfer; stimulated emission; amplified spontaneous emission; ultrafast spectroscopy 
The geometric confinement of graphene into few nanometers has allowed to open a finite band-gap in its electronic structure, which rendered the resulting nanographenes highly promising for a range of optoelectronic applications. ${ }^{1-3}$ Top-down approaches have been successfully used to fabricate nanographenes that are represented by quasi-1D graphene nanoribbons (GNRs), ${ }^{4}$ and quasi-0D graphene quantum dots (GQDs), ${ }^{5}$ although with certain degree of defects. On the other hand, bottomup synthesis has provided atomically precise GNRs ${ }^{6-7}$ and molecular GQDs. ${ }^{6,8-9}$ The latter approach has offered efficient control over energy gap and optical absorption/emission by tailoring the size and edge structures. ${ }^{6,10-13}$ Such bottom-up synthesized nanographenes are thus highly promising for applications in the field of nanotechnology, optics, and optoelectronics. ${ }^{14-15}$

Toward photonic applications, nanographene molecules have attracted a growing research interest very recently, owing to their optical absorption and luminescence properties that depend strongly on the degree of nanoconfinement and edge configuration. ${ }^{16-22}$ A large variety of GNRs and molecular GQDs with armchair edges have been synthesized and characterized by various methods, ${ }^{6}$, ${ }^{13}$ but there are still limited number of reports on nanographenes with zigzag edge although they generally exhibit intriguing properties, such as low energy gaps, biradical ground states character, and localized edge states. ${ }^{12,16-17,22-25}$ This is mainly due to the synthetic challenge in the incorporation of zigzag edges and their high environmental instability, which hampers their characterizations and utilization as functional materials. ${ }^{26}$ To this end, we have recently proposed dibenzo[hi,st]ovalene (DBOV) as a new molecular GQD with both armchair and zigzag edges, which displays remarkable stability, low energy gap, high oscillator strength, and a photoluminescence quantum yield (PLQY) as high as $79 \% .{ }^{27}$ Moreover, DBOV exhibits optical gain properties, amplified spontaneous emission (ASE) with a relatively low excitation threshold for organic emitters $\left(60 \mu \mathrm{J} \mathrm{cm}^{-2}\right),{ }^{27}$ and, interestingly, strong polaritonic emission at room temperature in microcavity. ${ }^{28}$

In our previous spectroscopic investigation on $\mathrm{DBOV},{ }^{27}$ we have noticed a relationship between the stability of the stimulated emission (SE) signal and intermolecular distance. Indeed, we have observed a dramatic and ultrafast (within $200 \mathrm{fs}$ ) quenching of SE when passing from solution (lifetime $\approx 300 \mathrm{ps}$ ) to solid films, whereas we were able to partially restore SE in diluted polymer matrix. We have tentatively attributed this effect to the formation of intermolecular polaronic-like species in solid film, whose absorption overlaps effectively with the SE band, in analogy with the photodynamic landscape depicted for conjugated polymers. ${ }^{29-31}$ Although the aggregation behavior as well as the charge transport properties in supramolecular adducts of graphene molecules, in particular hexa-peri-hexabenzocoronene derivatives, has been studied extensively, ${ }^{32}$ to date there are no detailed reports on the possible impact that charge generation in nanographenes can have on their optical properties. Such information can be highly relevant for the applications of nanographenes as 
emitters in light-emitting diodes and lasers, and as charge transport materials in solar cells and photodetectors.

Here, we report spectroscopic studies on DBOV derivatives with different substituents, providing evidence for strong competition between stimulated emission and charge generation in nanographenes. By systematically engineering the peripheral substituents, we found that the more substituted DBOV-Mes-C12 exhibits a sensibly slower SE deactivation dynamic (>1 ns) $)^{33}$ and lower ASE threshold in polystyrene (PS) matrix $\left(60 \mu \mathrm{J} \mathrm{cm}^{-2}\right)$ than the less functionalized DBOV-Mes (180 $\mu \mathrm{J} \mathrm{cm}^{-2}$ ) and DBOV-Ph (no ASE observed) (see Figure 1a for the structures). We attribute this effect to the higher tendency of the less functionalized DBOVs to form $\pi$-stacking aggregates with intermolecular charge transfer (CT) character, in which charges quench stimulated emission.

The structures of DBOV-Mes-C12, DBOV-Mes, and DBOV-Ph are displayed in Figure 1a and the synthesis was carried out through the method which we reported previously (see the SI). ${ }^{27-28}$ The peripheral substituents were varied to modify the propensity for aggregation: DBOV-Mes-C12 carries two bulky mesityl groups and two dodecyl chains and DBOV-Mes possesses only two mesityl groups while the DBOV-Ph has two smaller phenyl groups. The absorption spectra of these three derivatives in toluene (Figure 1b) show similar spectral features: i. a relatively strong peak at $626 \mathrm{~nm}$, $609 \mathrm{~nm}$ and $608 \mathrm{~nm}$ for DBOV-Mes-C12, DBOV-Mes, and DBOV-Ph, respectively, corresponding to the $0 \rightarrow 0$ ' electronic transition; ii. two weaker vibronic replica at 563-576 nm $\left(0 \rightarrow 1^{\prime}\right)$ and 513-520 $\mathrm{nm}\left(0 \rightarrow 2^{\prime}\right)$; iii. a broad absorption band peaked at 340-370 $\mathrm{nm}$ that can be attributed to a convolution of more energetic transitions (for the density functional theory calculations see S.I.). ${ }^{27-28}$

The lower $\mathrm{I}_{0-0} 0^{\prime} \mathrm{I}_{0-1}$, ratio of DBOV-Ph (2) than that of DBOV-Mes-C12 (2.8) and DBOVMes (2.7), and the appearance of a broad and red-shifted band for the less substituted molecules (DBOV-Mes and DBOV-Ph) (Figure 1b, inset), suggest the formation of stable $\pi$-stacked aggregates especially for the molecules lacking alkyl substitution. ${ }^{33-34} 35$ In addition, we embedded the three derivatives into a polystyrene (PS) matrix at $1 \mathrm{wt} \%$, a weight ratio that was already optimized for observing PL and ASE action in DBOV-Mes-C12. ${ }^{27}$ The optical absorption of the films (Figure 1c) shows regular fringes that can be attributed to interference phenomena (see Figure $\mathbf{S 4}$ for the reflection spectra), which in-fact hinder a detailed UV-VIS absorption characterization of the diluted blends in polymer matrix. On the other hand, the PLE spectra (Figure 1d and 1e for solutions in toluene and PS films) are a more sensitive probe for discriminating the different behavior of the three derivatives. In the toluene solutions, a larger FWHM is observed for DBOV-Mes (39 nm) and DBOV-Ph $(36 \mathrm{~nm})$ compared to that of DBOV-Mes-C12 $(24 \mathrm{~nm})$, together with relatively higher PLE signal in the UV region (350-400 nm) when normalized at the PLE maxima. Furthermore, a decreased $\mathrm{I}_{0-0}, \mathrm{I}_{0-1}$, ratio is revealed for DBOV-Mes (1.2) and DBOV-Ph (1.3) in comparison with 
DBOV-Mes-C12 (2). All these point toward a stronger tendency for the least substituted molecules to establish dipolar intermolecular interactions and form $\pi$-stacking aggregates. Although the narrower FWHM of the 0-0' PLE peaks in PS matrix (18 $\mathrm{nm}$ for the three molecules) indicates a larger intermolecular distance than in the $0.1 \mathrm{mg} / \mathrm{mL}$ solutions, the higher PLE signal in the 350-400 $\mathrm{nm}$ region for DBOV-Mes and especially for DBOV-Ph can be attributed to the partial retaining of aggregates also in the relatively diluted solid matrix. Although we observe some of the typical spectroscopic signatures of $\mathrm{H}$-aggregation for these nanographenes, further experiments are currently underway to elucidate the exact nature of this phenomenon. 
a

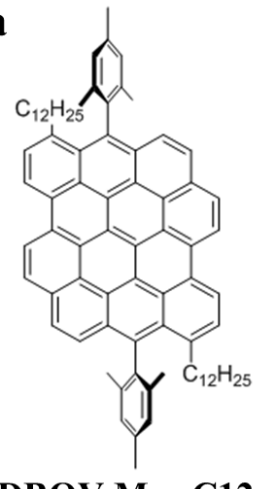

DBOV-Mes-C12

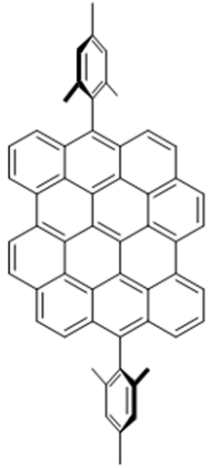

DBOV-Mes

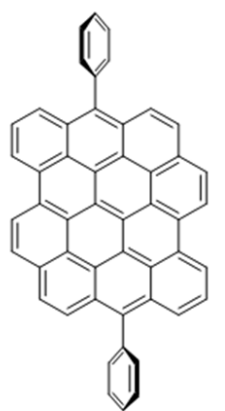

DBOV-Ph b

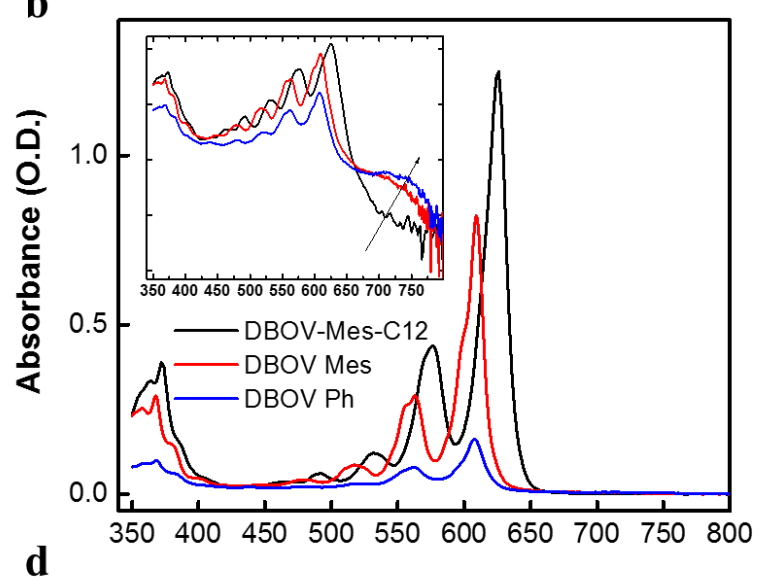

d

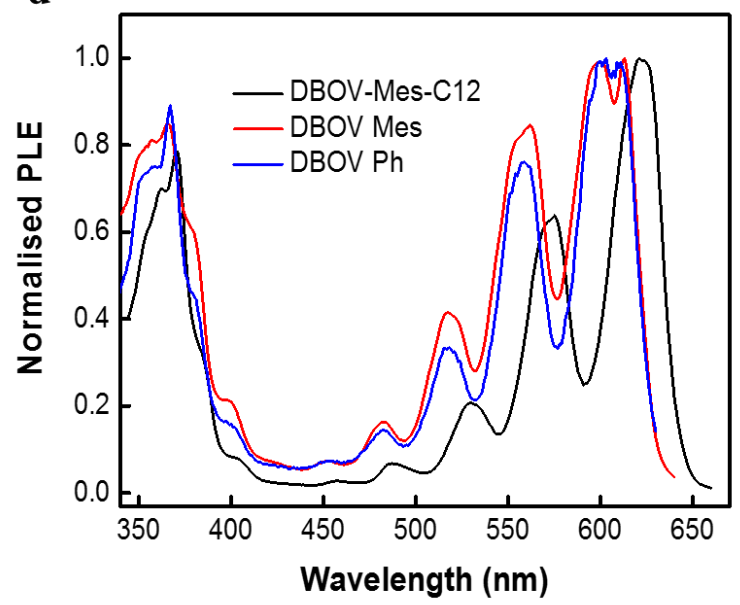

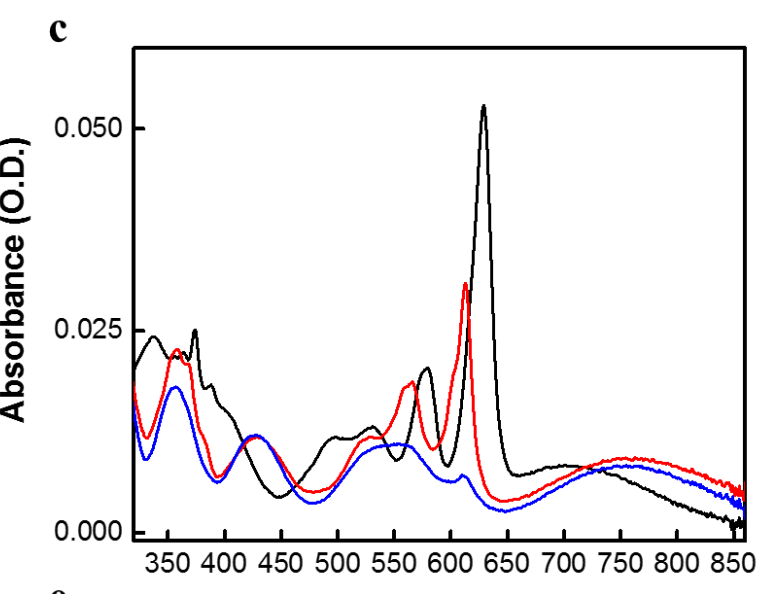

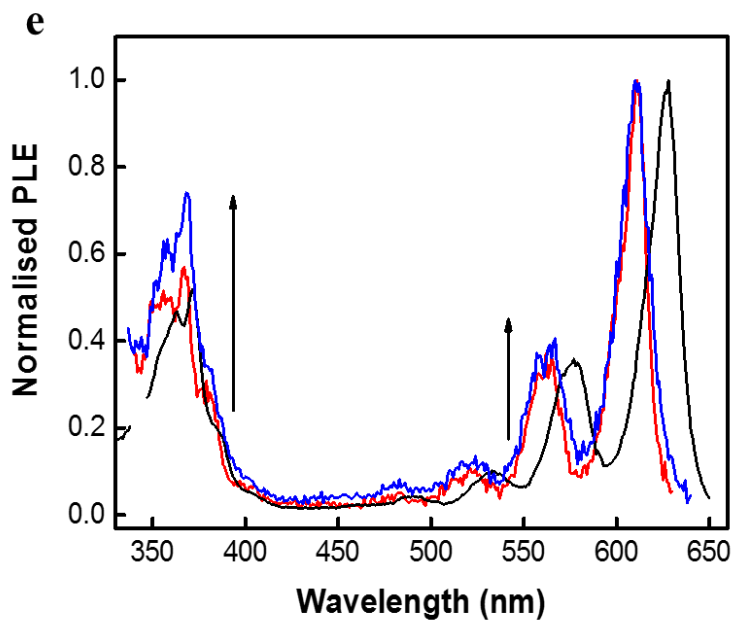

Figure 1. (a) Molecular sketches of the three DBOV derivatives. (b,c) UV-VIS absorption spectra of the three derivatives (b) in toluene solution $(0.1 \mathrm{mg} / \mathrm{ml})$ and (c) in PS matrix (DBOV:PS 1wt \% ratio). (d,e) PLE spectra of the DBOV derivatives (d) in toluene solution $(0.1 \mathrm{mg} / \mathrm{ml})$ and (e) in PS matrix (DBOV:PS $1 \mathrm{wt} \%$ ratio).

In Figure 2 we present the differential transmission spectra and dynamics in toluene solution $(0.1 \mathrm{mg} / \mathrm{mL})$ and PS matrix. The transient spectra of the DBOV molecules (Figure $2 \mathrm{a}, \mathrm{b}, \mathrm{d}, \mathrm{e}, \mathrm{g}, \mathrm{h}$, display three main peaks, namely: i. a negative feature at around $500 \mathrm{~nm}$ that can be linked to photoinduced absorption (PA) from the first excited state $\left(S_{1}\right)$ to higher lying states $\left(S_{n}\right)$; ii. the main peak centered at $625 \mathrm{~nm}$ for DBO1 and $610 \mathrm{~nm}$ for DBOV-Mes and DBOV-Ph, can be attributed to 
the depletion of the ground state due to the $0 \rightarrow 0$ ' transition (photobleaching PB); iii. a side positive peak at 560-570 $\mathrm{nm}$ corresponding to its vibronic replica $\left(0 \rightarrow 1^{\prime}\right)$; iv. a positive peak at $670-680 \mathrm{~nm}$ that can be associated to the stimulated emission signal (SE). ${ }^{27}$ The spectra of the three molecules exhibit an intriguing behavior as a function of pump-probe delay. In particular, we observe the complete suppression of the SE and its incorporation into a large negative PA feature (650-720 nm), as well as the appearance of a well-defined derivative-shaped peak resembling an electroabsorption signal. Such an effect seems to be clearer in the less substituted molecules, and especially for the least substituted DBOV-Ph for which we see a progressive build-up of the electroabsorption-like signal. Conversely, if we either diluted the solutions down to $0.01 \mathrm{mg} / \mathrm{mL}$ (Figure S5) or embedded the molecules in a diluted PS matrix (Figures 2b,e,h), both the PA signal and the delayed electroabsorption are suppressed. Such behavior is thus ultimately related to the intermolecular distance experienced by the nanographenes. Therefore, we preliminary assign the derivative shape signal to a Stark shift due to charge formation, whereas the PA signal can be connected to charge absorption. ${ }^{29}$ Note that we could not observe such effect in the previous study on DBOV-Mes-C12, ${ }^{27}$ as we used a lower concentration $(0.05 \mathrm{mg} / \mathrm{mL})$ than in the work reported here. The delayed appearance of these two peaks can be linked to depopulation of the excited states (decay of the PB and SE signals) followed by the rise of the charge state. Furthermore, we reckon that one single nanographene molecule cannot sustain the formation of a stable charge population, therefore these effects occur most likely in intermolecular adducts (i.e. dimers, trimers), rather than in one single nanographene unit only. These hypotheses are corroborated by the fact that the less substituted DBOV-Ph, which possesses the highest tendency to form aggregates as indicated by the steady-state absorption and PLE data, exhibits the best-defined electroabsorption signal among the three molecules.

If we pass to the transient dynamics of the stimulated emission signal (Figure $2 \mathrm{e}, \mathrm{f}$,) that is of great interest for applications of these molecules in photonic devices, we can detect a clear connection between the degree of functionalization and the photodynamic. Starting from DBOV-Mes-C12, the SE decay rate follows nicely the dilution series, as the signal lifetime is $400 \mathrm{ps}$ for the $0.1 \mathrm{mg} / \mathrm{mL}$ solution and $1 \mathrm{~ns}$ for the PS film, whereas it does not decay at all for the $0.01 \mathrm{mg} / \mathrm{mL}$ solution (see table 1 for the SE lifetimes). This can be explained in terms of the relatively high solubility of this derivative containing two dodecyl chains, which allows one to tune easily the degree of aggregation. DBOV-Mes displays a general decrease of the lifetime if compared to DBOV-Mes-C12, as well as a virtually identical photodynamic between the 0.1 and $0.01 \mathrm{mg} / \mathrm{mL}$ solutions. It is worth to note a hint of an initial ultrafast decay for this molecule that in principle can be a signature of ultrafast charge transfer processes (<100 fs), which unfortunately we could not resolve with the temporal resolution 
of our experiment (150 fs). Finally, DBOV-Ph solutions exhibit further lower lifetimes than the other derivatives and, despite the noise signal, in this case we can clearly discriminate an initial decay for the DBOV-Ph film, which loses $\approx 60 \%$ of the population in 200 fs. This fast decay can be due to ultrafast charge transfer processes in the DBOV-Ph aggregates still present in PS dilute matrix, whereas the long decay time ( $1 \mathrm{~ns})$ can be related to hole bleaching. These effects most probably arise from the poorer solubility of DBOV-Ph than that of the other molecules, both in solution and polymer matrix. We can thus conclude that the SE deactivation rate increases with decreasing bulkiness of the substituents, an effect that can be ascribed to an augmented tendency for the least substituted molecules to form aggregates with charge transfer character, in which charge generation prevails over SE.

$\mathbf{a}$
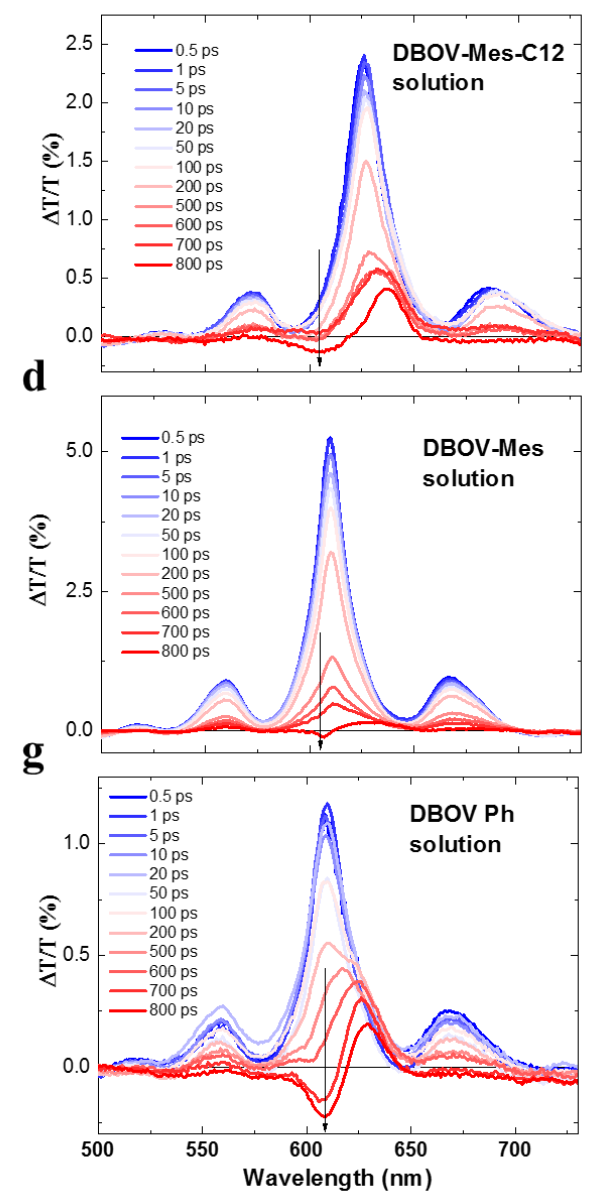

b

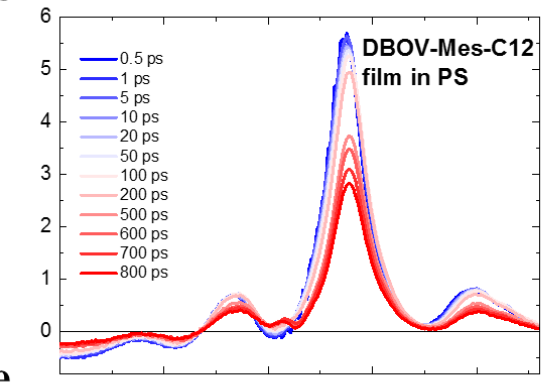

e
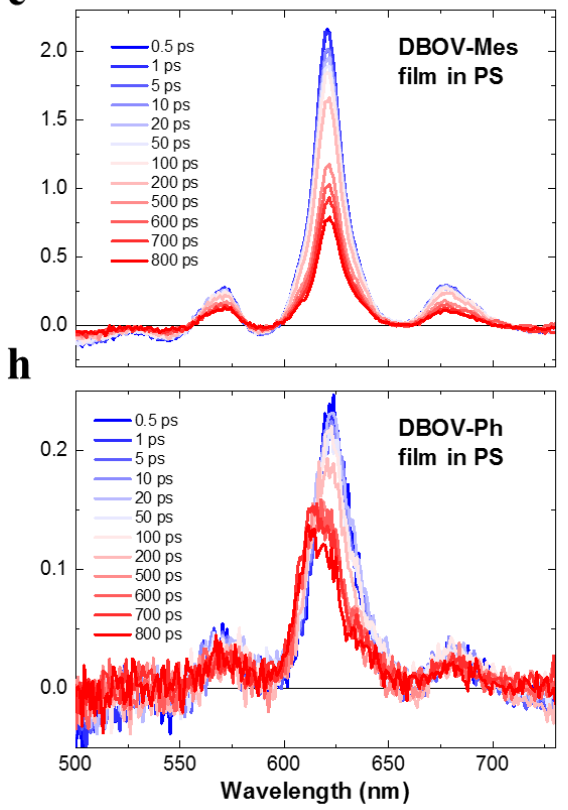

c

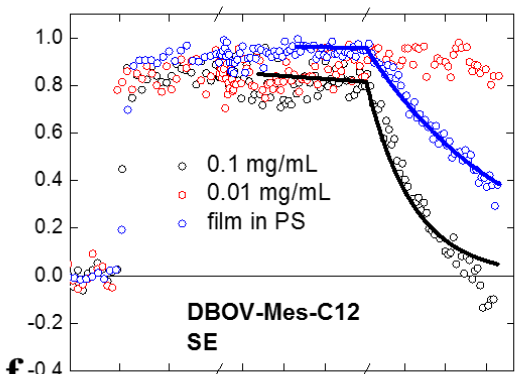

f- -0

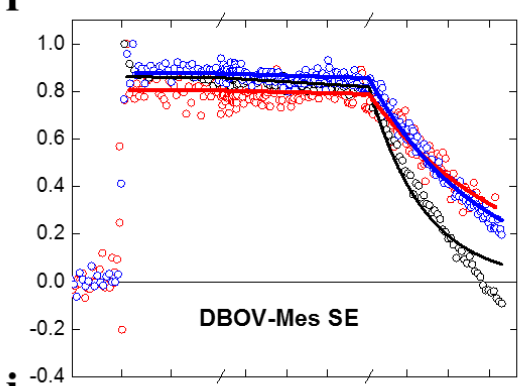

$\mathbf{i}^{-0.4}$

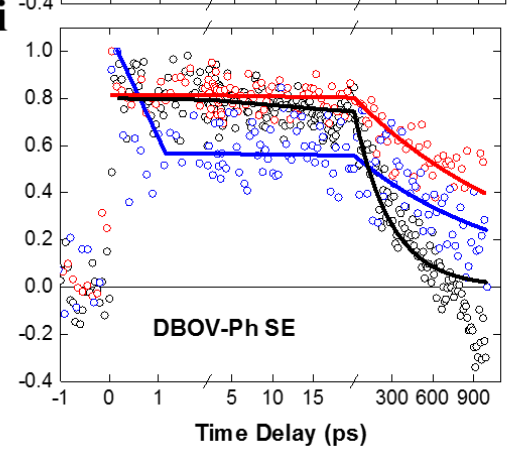

Figure 2. (a,d,g) Transient absorption spectra as a function of pump-probe delay for DBOV-MES-C12, DBOV Mes and DBOV Ph in toluene solution $(0.1 \mathrm{mg} / \mathrm{mL})$ and $(\mathrm{b}, \mathrm{e}, \mathrm{h})$ in PS matrix. (c,f,i) Transient dynamics for the three derivatives till $1 \mathrm{~ns}$. We used an excitation wavelength of $600 \mathrm{~nm}$ for the solutions in toluene and $610 \mathrm{~nm}$ for the films in PS. 
Table 1. Calculated lifetimes of the SE signal for DBOV-MES-C12, DBOV Mes and DBOV Ph.

\begin{tabular}{llll}
\hline SE lifetime & $\mathbf{0 . 1}$ & $\mathbf{0 . 0 1} \mathbf{~ m g / m L}$ & Film in PS \\
& $\mathbf{m g} / \mathbf{m L}$ & & \\
\hline DBOV-Mes-C12 & $400 \mathrm{ps}$ & Long-living & $>1 \mathrm{~ns}$ \\
DBOV Mes & $400 \mathrm{ps}$ & $1 \mathrm{~ns}$ & $1 \mathrm{~ns}$ \\
DBOV Ph & $270 \mathrm{ps}$ & $1 \mathrm{~ns}$ & $\tau_{1}<100 \mathrm{fs}$ \\
& & & $\tau_{2} \approx 1 \mathrm{~ns}$ \\
\hline
\end{tabular}

Finally, to gain further insights into the effect of functionalization on the optical gain properties, we performed ASE measurements on the solid films. We used a PS : nanographene $1 \mathrm{wt} \%$ , a ratio that we have already optimized in previous ASE experiments. ${ }^{27}$ In particular, we have seen that such a concentration allows to minimize the occurrence of intermolecular charge transfer processes that would suppress gain, while maintaining a sufficient amount of material necessary for waveguiding the excitation and achieving ASE action. In agreement with the abovementioned findings, we observe a three-fold increase of the ASE threshold for the DBOV-Mes $\left(180 \mu \mathrm{J} / \mathrm{cm}^{2}\right)$ than for DBOV-Mes-C12 $\left(60 \mu \mathrm{J} / \mathrm{cm}^{2}\right)$, whereas we could not observe any ASE action for DBOVPh. This indicates that at the same concentration the three molecules experience three different intermolecular environments which strongly depend on the presence of substituents acting as intermolecular spacers. For instance, the lack of substitution in DBOV-Ph leads to a stronger tendency to aggregation not only in solution, but also in the diluted polymer matrix. In these aggregates, gain and photoluminescence are quenched due to the strong competition with ultrafast charge transfer phenomena, as it has been shown in the TA section. On the other hand, although we can still observe ASE action for DBVO-Mes, the fluency threshold to achieve the ASE regime is higher than for DBOV-Mes-C12, as a result of a closer intermolecular distance in this molecule as compared with the dodecyl substituted case. 
a

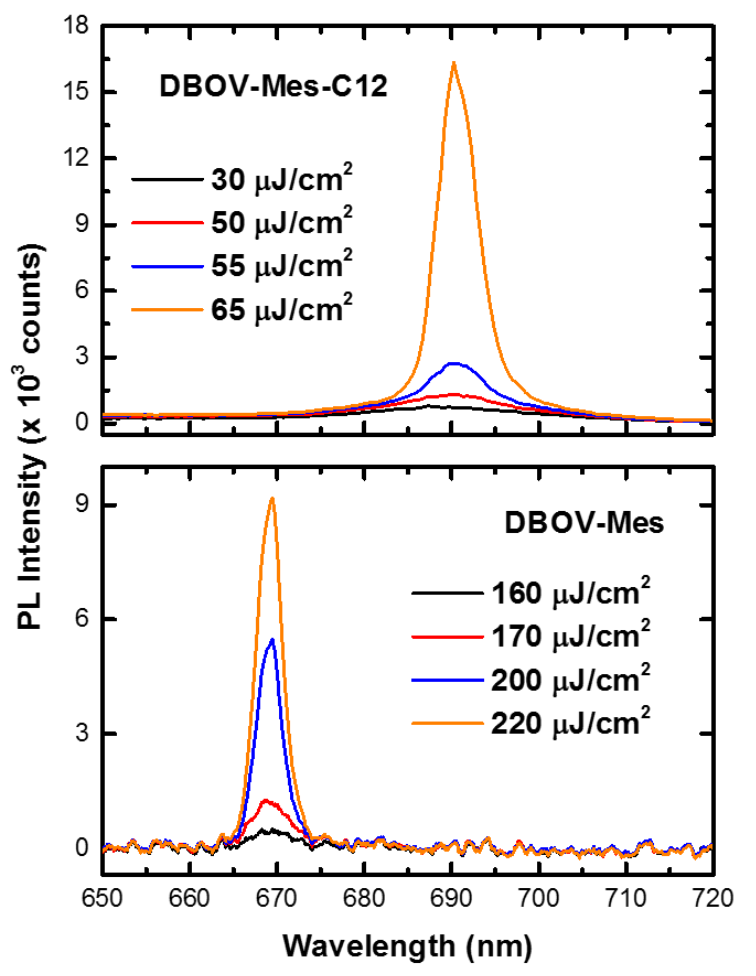

b

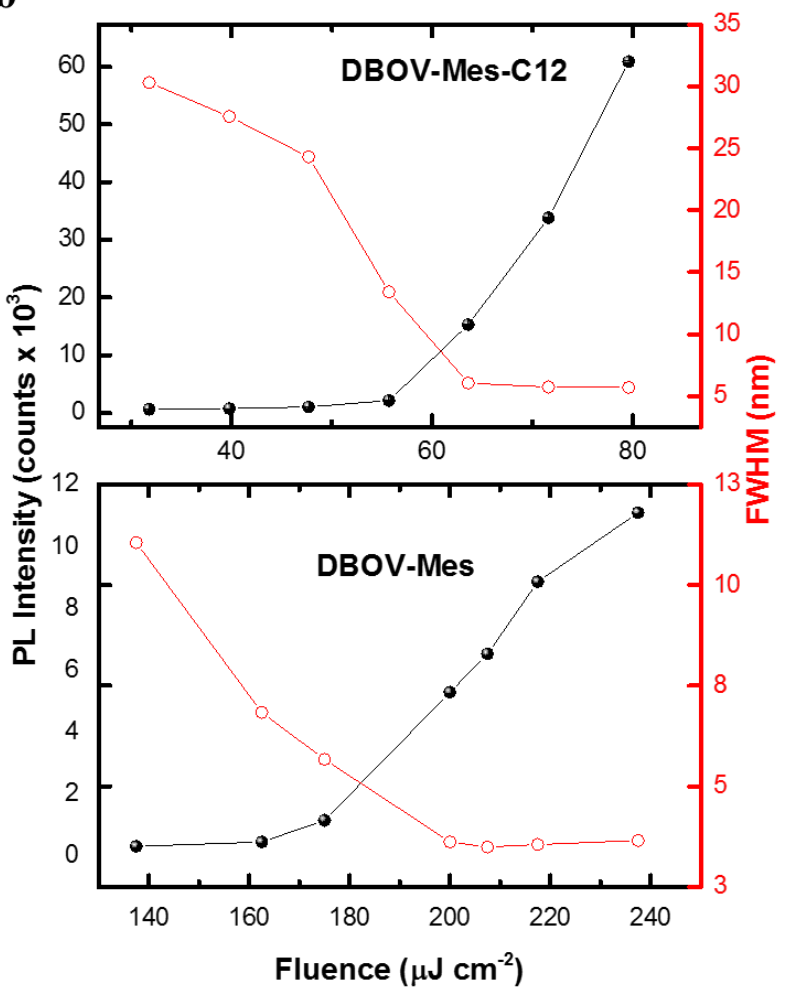

Figure 3. (a) Photoluminescence spectra taken by exciting at $620 \mathrm{~nm}$ (DBOV-Mes-C12, top graph) and $610 \mathrm{~nm}$ (DBOV-Mes, bottom graph) the films in PS (1wt. \%) with a femtosecond laser. (b) Input-output characteristics of ASE action for the $1 \mathrm{wt} \%$ blend in PS for DBOV-Mes-C12 (top graph) and DBOV-Mes (bottom graph).

In conclusion, we have investigated the effect of peripheral functionalization on the nonlinear optical properties of three structurally defined nanographenes. Our results indicate a strong competition between optical gain and charge generation. The latter process possesses intermolecular character and thus prevails in concentrated solution and, in general, in those molecules inhibits adequate separation between the electronically active conjugated cores. For these reasons, the less substituted DBOV-Mes and especially DBOV-Ph display shorter SE lifetimes than DBOV-MesC12 bearing two additional dodecyl groups. ASE measurements corroborate this scenario, as the excitation fluency threshold for achieving ASE action is three times higher in DBOV-Mes than DBOV-Mes-C12, while DBOV-Ph does not show any ASE. These findings can be important for selectively favoring either the light emitting or charge transport properties by simply engineering their side-group functionalities. Further studies into the photonics (i.e. lasers) and optoelectronics (light emitting diodes and solar cells) of large nanographene molecules are thus highly promising. 


\section{Acknowledgements}

We thank the financial support from the EU Horizon 2020 Research and Innovation Programme under Grant Agreement N. 643238 (SYNCHRONICS) and the Max Planck Society.

\section{References}

1. Bao, Q.; Loh, K. P. Graphene photonics, plasmonics, and broadband optoelectronic devices. ACS Nano 2012, 6 (5), 3677-94.

2. Loh, K. P.; Tong, S. W.; Wu, J. Graphene and Graphene-like Molecules: Prospects in Solar Cells. $J$ Am Chem Soc 2016, 138 (4), 1095-102.

3. Li, L.; Wu, G.; Yang, G.; Peng, J.; Zhao, J.; Zhu, J. J. Focusing on luminescent graphene quantum dots: current status and future perspectives. Nanoscale 2013, 5 (10), 4015-39.

4. $\mathrm{Xu}, \mathrm{W}$. T.; Lee, T. W. Recent progress in fabrication techniques of graphene nanoribbons. Materials Horizons 2016, 3 (3), 186-207.

5. Bacon, M.; Bradley, S. J.; Nann, T. Graphene Quantum Dots. Particle \& Particle Systems Characterization 2014, 31 (4), 415-428.

6. Narita, A.; Wang, X. Y.; Feng, X.; Müllen, K. New advances in nanographene chemistry. Chem Soc Rev 2015, 44 (18), 6616-43.

7. Talirz, L.; Ruffieux, P.; Fasel, R. On-Surface Synthesis of Atomically Precise Graphene Nanoribbons. Advanced materials 2016, 28 (29), 6222-31.

8. Yan, X.; Li, B.; Li, L. S. Colloidal graphene quantum dots with well-defined structures. Acc Chem Res 2013, 46 (10), 2254-62.

9. Chen, L.; Hernandez, Y.; Feng, X.; Mullen, K. From nanographene and graphene nanoribbons to graphene sheets: chemical synthesis. Angewandte Chemie 2012, 51 (31), 7640-54.

10. $\quad$ Tan, Y. Z.; Yang, B.; Parvez, K.; Narita, A.; Osella, S.; Beljonne, D.; Feng, X.; Mullen, K. Atomically precise edge chlorination of nanographenes and its application in graphene nanoribbons. Nat Commun 2013, 4, 2646.

11. Wu, J.; Pisula, W.; Müllen, K. Graphenes as Potential Material for Electronics. Chemical Reviews 2007, 107 (3), 718-747.

12. Segawa, Y.; Ito, H.; Itami, K. Structurally uniform and atomically precise carbon nanostructures. Nature Reviews Materials 2016, 1 (1), 15002.

13. Wang, X.-Y.; Narita, A.; Müllen, K. Precision synthesis versus bulk-scale fabrication of graphenes. Nature Reviews Chemistry 2017, 2 (1), 0100.

14. Osella, S.; Narita, A.; Schwab, M. G.; Hernandez, Y.; Feng, X.; Mullen, K.; Beljonne, D. Graphene nanoribbons as low band gap donor materials for organic photovoltaics: quantum chemical aided design. ACS Nano 2012, 6 (6), 5539-48.

15. Chen, Z.; Wang, H. I.; Teyssandier, J.; Mali, K. S.; Dumslaff, T.; Ivanov, I.; Zhang, W.; Ruffieux, P.; Fasel, R.; Rader, H. J.; Turchinovich, D.; De Feyter, S.; Feng, X.; Klaui, M.; Narita, A.; Bonn, M.; Mullen, K. Chemical Vapor Deposition Synthesis and Terahertz Photoconductivity of Low-Band-Gap N = 9 Armchair Graphene Nanoribbons. J Am Chem Soc 2017, 139 (10), 3635-3638.

16. Hayashi, H.; Aratani, N.; Yamada, H. Semiconducting Self-Assembled Nanofibers Prepared from Photostable Octafluorinated Bisanthene Derivatives. Chemistry 2017, 23 (29), 7000-7008.

17. Wu, J.; Gu, Y.; Wu, X.; Gopalakrishna, T. Y.; Phan, H. Graphene - like Molecules with Four Zigzag Edges. Angewandte Chemie 2018.

18. Soavi, G.; Dal Conte, S.; Manzoni, C.; Viola, D.; Narita, A.; Hu, Y.; Feng, X.; Hohenester, U.; Molinari, E.; Prezzi, D.; Mullen, K.; Cerullo, G. Exciton-exciton annihilation and biexciton stimulated emission in graphene nanoribbons. Nat Commun 2016, 7, 11010. 
19. Zhu, S.; Wang, L.; Li, B.; Song, Y.; Zhao, X.; Zhang, G.; Zhang, S.; Lu, S.; Zhang, J.; Wang, H.; Sun, H.; Yang, B. Investigation of photoluminescence mechanism of graphene quantum dots and evaluation of their assembly into polymer dots. Carbon 2014, 77, 462-472.

20. Wang, X. Y.; Narita, A.; Zhang, W.; Feng, X.; Mullen, K. Synthesis of Stable Nanographenes with OBO-Doped Zigzag Edges Based on Tandem Demethylation-Electrophilic Borylation. J Am Chem Soc 2016, 138 (29), 9021-4.

21. Zhao, S.; Rondin, L.; Delport, G.; Voisin, C.; Beser, U.; Hu, Y.; Feng, X.; Mullen, K.; Narita, A.; Campidelli, S.; Lauret, J. S. Fluorescence from graphene nanoribbons of well-defined structure. Carbon 2017, 119, 235-240.

22. Ajayakumar, M. R.; Fu, Y.; Ma, J.; Hennersdorf, F.; Komber, H.; Weigand, J. J.; Alfonsov, A.; Popov, A. A.; Berger, R.; Liu, J.; Mullen, K.; Feng, X. Toward Full Zigzag-Edged Nanographenes: periTetracene and Its Corresponding Circumanthracene. J Am Chem Soc 2018, 140 (20), 6240-6244.

23. Ruffieux, P.; Wang, S.; Yang, B.; Sanchez-Sanchez, C.; Liu, J.; Dienel, T.; Talirz, L.; Shinde, P.; Pignedoli, C. A.; Passerone, D.; Dumslaff, T.; Feng, X.; Mullen, K.; Fasel, R. On-surface synthesis of graphene nanoribbons with zigzag edge topology. Nature 2016, 531 (7595), 489-92.

24. Konishi, A.; Hirao, Y.; Matsumoto, K.; Kurata, H.; Kishi, R.; Shigeta, Y.; Nakano, M.; Tokunaga, K.; Kamada, K.; Kubo, T. Synthesis and characterization of quarteranthene: elucidating the characteristics of the edge state of graphene nanoribbons at the molecular level. J Am Chem Soc 2013, 135 (4), 1430-7.

25. Sun, Z.; Zeng, Z.; Wu, J. Zethrenes, extended p-quinodimethanes, and periacenes with a singlet biradical ground state. Acc Chem Res 2014, 47 (8), 2582-91.

26. Liu, J.; Ravat, P.; Wagner, M.; Baumgarten, M.; Feng, X.; Müllen, K. Tetrabenzo[a,f,j,o]perylene: a polycyclic aromatic hydrocarbon with an open-shell singlet biradical ground state. Angewandte Chemie 2015, 54 (42), 12442-6.

27. Paterno, G. M.; Chen, Q.; Wang, X. Y.; Liu, J.; Motti, S. G.; Petrozza, A.; Feng, X.; Lanzani, G.; Mullen, K.; Narita, A.; Scotognella, F. Synthesis of Dibenzo[hi,st]ovalene and Its Amplified Spontaneous Emission in a Polystyrene Matrix. Angewandte Chemie 2017, 56 (24), 6753-6757.

28. Coles, D. M.; Chen, Q.; Flatten, L. C.; Smith, J. M.; Mullen, K.; Narita, A.; Lidzey, D. G. Strong Exciton-Photon Coupling in a Nanographene Filled Microcavity. Nano Lett 2017, 17 (9), 5521-5525.

29. Cabanillas-Gonzalez, J.; Grancini, G.; Lanzani, G. Pump-probe spectroscopy in organic semiconductors: monitoring fundamental processes of relevance in optoelectronics. Advanced materials 2011, 23 (46), 5468-85.

30. Yan, M.; Rothberg, L. J.; Kwock, E. W.; Miller, T. M. Interchain Excitations in Conjugated Polymers. Phys Rev Lett 1995, 75 (10), 1992-1995.

31. Virgili, T.; Marinotto, D.; Lanzani, G.; Bradley, D. D. C. Ultrafast resonant optical switching in isolated polyfluorenes chains. Applied Physics Letters 2005, 86 (9), 91113.

32. Pisula, W.; Feng, X.; Müllen, K. Charge-Carrier Transporting Graphene-Type Molecules†. Chemistry of Materials 2011, 23 (3), 554-567.

33. Kastler, M.; Pisula, W.; Wasserfallen, D.; Pakula, T.; Mullen, K. Influence of alkyl substituents on the solution- and surface-organization of hexa-peri-hexabenzocoronenes. J Am Chem Soc 2005, 127 (12), 4286-96.

34. Feng, X.; Pisula, W.; Zhi, L.; Takase, M.; Mullen, K. Controlling the columnar orientation of C3symmetric "superbenzenes" through alternating polar/apolar substitutents. Angewandte Chemie 2008, 47 (9), 1703-6.

35. Spano, F. C. The spectral signatures of Frenkel polarons in $\mathrm{H}-$ and J-aggregates. Acc Chem Res 2010, 43 (3), 429-39. 


\section{Supporting Information for \\ Modulation of the Nonlinear Optical Properties of Dibenzo[hi,st]ovalene by Engineering of Peripheral Substituents}

\section{Experimental details}

\section{General:}

All reactions dealing with air- or moisture-sensitive compounds were carried out in a dry reaction vessel under argon. Analytical thin layer chromatography (TLC) was performed on silica gel coated substrates " $60 \mathrm{~F}_{254}$ " from Merck. Preparative column chromatography was performed on silica gel from Merck with a grain size of $0.063-0.200 \mathrm{~mm}$ (silica gel) or $0.040-0.063 \mathrm{~mm}$ (flash silica gel). High-resolution mass spectra (HRMS) were recorded by matrix-assisted laser decomposition/ionization (MALDI) using 7,7,8,8-tetracyanoquinodimethane (TCNQ) as matrix on a Bruker Reflex II-TOF spectrometer. Raman spectra were recorded with a Bruker RFS 100/S Raman spectrometer excited by a $488 \mathrm{~nm}$ laser. FT-IR spectra were obtained using a FT-IR spectrophotometer TENSOE II Brucker equipped with platinum attenuated total reflection (ATR) in the range between 400 and $4000 \mathrm{~cm}^{-1}$ with a resolution of $4 \mathrm{~cm}^{-1}$. An average of 64 scans has been taken for each sample.

\section{Materials:}

Benzo[ $[a]$ dinaphtho[2,1,8-cde:1',2',3',4'-ghi]perylene-5,14-dicarbaldehyde (1), DBOV-Mes-C12, and DBOV-Mes were synthesized according to the methods described in our previous reports. ${ }^{1,2}$ All other chemicals were purchased from commercial sources and used without further purification.

\section{Synthesis of DBOV-Ph.}

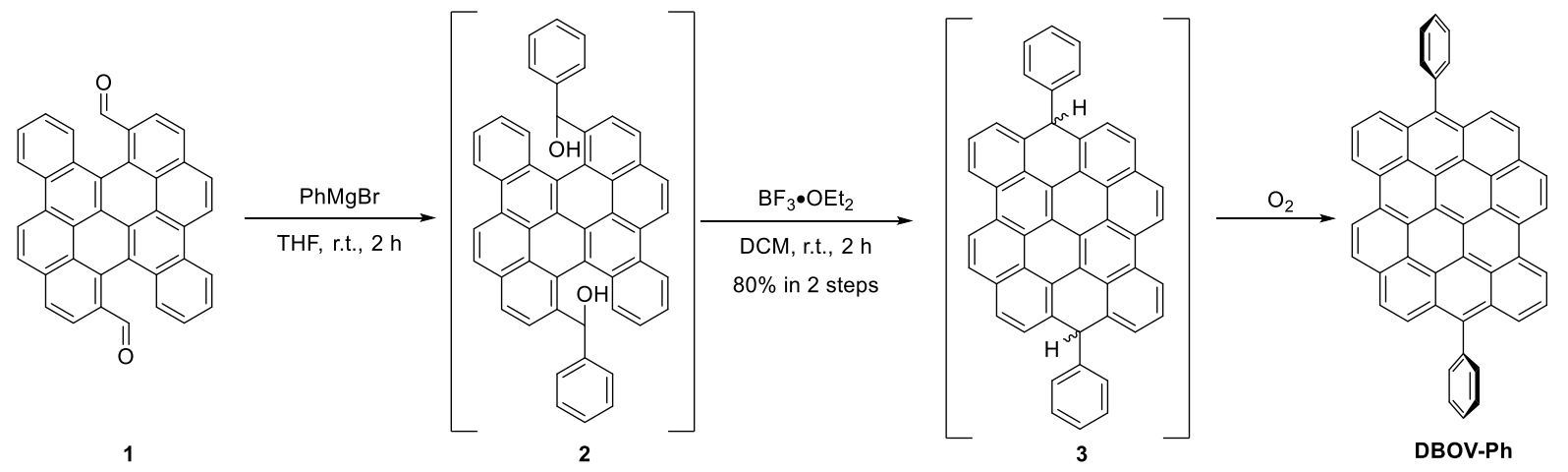

Scheme S1. Synthetic route towards DBOV-Ph.

To a solution of benzo[a]dinaphtho[2,1,8-cde:1',2',3',4'-ghi]perylene-5,14-dicarbaldehyde (1) ${ }^{\mathbf{1}}$ (5.0 $\mathrm{mg}, 9.9 \mathrm{mmol})$ dissolved in anhydrous tetrahydrofuran $(5 \mathrm{~mL})$ was added $\mathrm{PhMgBr}(0.080 \mathrm{~mL}, 0.16$ 
mmol, 2.0 $\mathrm{M}$ in tetrahydrofuran). After stirring at room temperature for $2 \mathrm{~h}$, the reaction was quenched by addition of saturated aqueous solution of $\mathrm{NH}_{4} \mathrm{Cl}(10 \mathrm{~mL})$ and extracted with ethyl acetate $(10 \mathrm{~mL})$ for three times. The combined organic phases were washed with brine, dried over $\mathrm{Na}_{2} \mathrm{SO}_{4}$, and evaporated to give an orange solid, which was used for the next step without further purification. The obtained solid was dried under vacuum for $2 \mathrm{~h}$ and dissolved in anhydrous dichloromethane (5 $\mathrm{mL})$. After addition of $\mathrm{BF}_{3} \cdot \mathrm{OEt}_{2}(0.5 \mathrm{~mL})$, the solution was stirred at room temperature for $2 \mathrm{~h}$ under air. Methanol $(20 \mathrm{~mL})$ was then added and the suspension was filtered, followed by washing with dichloromethane $(50 \mathrm{~mL})$ to give the title compound $(5 \mathrm{mg}, 80 \%$ yield) as blue solid. Measurement of ${ }^{1} \mathrm{H}$ NMR was not possible even at $140{ }^{\circ} \mathrm{C}$ in $o$-dichlorobenzene $\left(d_{4}\right)$ most probably because of the low solubility and strong aggregation in organic solvents. HRMS (MALDI-TOF, positive) $\mathrm{m} / \mathrm{z}$ : calcd. for $\mathrm{C}_{50} \mathrm{H}_{24}{ }^{+}(\mathrm{M})^{+}$: 624.1873; found: 624.1859 (error $=2 \mathrm{ppm}$ ). Additional structural evidences could be obtained by comparison of FTIR, Raman, UV-VIS absorption, and PLE spectra with those of DBOV-Mes, which could be fully characterized by NMR in our previous report (see Figure 1 and S6). ${ }^{1}$

Steady-state absorption and photoluminescence excitation. For the UV-VIS absorption measurements, we used a Perkin Elmer Lambda 1050 spectrophotometer, equipped with deuterium $(180-320 \mathrm{~nm})$ and tungsten (320-3300 nm) lamps and a photomultiplier detector (180-860 nm. All the absorption spectra were corrected for the reference spectra taken at $100 \%$ transmission (without the sample) at $0 \%$ transmission (with an internal attenuator), and for the background spectrum (toluene only or glass). The PLE spectra were taken with a Horiba Nanolog Fluorimeter, equipped with a xenon lamp, two monochromators and two detectors (photomultiplier and InGaAs).

Ultrafast pump-probe spectroscopy. For the non-degenerate pump and probe measurements, the molecules were dissolved in toluene with a concentration of 0.1 and $0.01 \mathrm{mg} \mathrm{mL}^{-1}$. For DBOV: polystyrene solid blends ( $1 \%$ weight ratio), we dissolved the proper amount of material in a $40 \mathrm{mg} / \mathrm{mL}$ polystyrene solution in toluene (PS, Aldrich, $\mathrm{Mw}=200,000$ ). Then, the blends were spin-cast onto a glass substrate with a spin speed of 1000 rotations per minute yielding a thickness of $\approx 400 \mathrm{~nm}$, as measured by profilometer. We employed an amplified Ti:sapphire laser with $2 \mathrm{~mJ}$ output energy, 1 $\mathrm{kHz}$ repetition rate and a central energy of $1.59 \mathrm{eV}(800 \mathrm{~nm})$. We used a pump wavelength of 610 $\mathrm{nm}$, which is resonant with the main $\pi \rightarrow \pi^{*}$ transition. Such pump pulses were generated by using a visible optical parameter amplifier (OPA). Pump pulses were focused on a $200 \mu \mathrm{m}$ spot (diameter), keeping pump fluences at $\sim 50 \mu \mathrm{J} \mathrm{cm}-2$. As a probe pulse, we used a broadband white light supercontinuum generated in a sapphire plate from $450 \mathrm{~nm}$ to $780 \mathrm{~nm}$.

Amplified spontaneous emission measurements. For ASE measurements, we employed the same films produced for pump-probe measurements. ASE characterizations were performed by using an amplified Ti:sapphire laser with $2 \mathrm{~mJ}$ output energy and $2 \mathrm{kHz}$ repetition rate at $625 \mathrm{~nm}$ for DBOVMes-C12 and $610 \mathrm{~nm}$ for DBOV-Mes and DBOV-Ph. We used a $7.5 \mathrm{~cm}$ cylindrical focal lens to focus the pump beam into a $2 \mathrm{~mm} \times 0.1 \mathrm{~mm}$ strip and collected the emission from the edge of the film with a fibre spectrometer (resolution $0.5 \mathrm{~nm}$ ).

\section{Density functional theory calculations}


Optimized geometries and simulated spectra. The DBOV molecules have been sketched with the Avogadro package. ${ }^{3}$ The optimization of the ground state geometry and the calculation of the electronic transitions have been performed with the package ORCA $3.0 .3,{ }^{4}$ using the B3LYP functional ${ }^{5}$ in the framework of the density functional theory. The Ahlrichs split valence basis set ${ }^{6}$ and the all-electron nonrelativistic basis set SVPalls $1^{7,8}$ have been employed. Moreover, the calculation utilizes the Libint library. ${ }^{9}$

\section{Optimized geometries}

Figure S1. Optimized geometries of DBOV-Mes-C12. 
Figure S2. Optimized geometries of DBOV-Mes.

Figure S3. Optimized geometries of DBOV-Ph. 


\begin{tabular}{|c|c|c|}
\hline \multicolumn{3}{|c|}{ Absorption spectra } \\
\hline \multicolumn{3}{|c|}{ DBOV-Mes-C12 } \\
\hline State & $\begin{array}{l}\text { Wavelength } \\
(\mathrm{nm})\end{array}$ & $\begin{array}{l}\text { Oscillator } \\
\text { strength }\end{array}$ \\
\hline 1 & 653.7 & 1.402 .510 .039 \\
\hline 2 & 540.3 & 0.007853140 \\
\hline 3 & 480.5 & 0.022907590 \\
\hline 4 & 473.2 & 0.014948088 \\
\hline 5 & 451.0 & 0.001972269 \\
\hline 6 & 427.3 & 0.010790536 \\
\hline 7 & 410.6 & 0.006305509 \\
\hline 8 & 399.5 & 0.151176553 \\
\hline 9 & 397.1 & 0.004598530 \\
\hline 10 & 392.5 & 0.049129966 \\
\hline 11 & 388.3 & 0.050020031 \\
\hline 12 & 374.1 & 0.102582346 \\
\hline 13 & 365.8 & 0.000944822 \\
\hline 14 & 364.5 & 0.041416256 \\
\hline 15 & 358.2 & 0.021301294 \\
\hline 16 & 341.4 & 0.022966732 \\
\hline
\end{tabular}

\section{DBOV-Mes}

$\begin{array}{ccc}\text { State } & \begin{array}{c}\text { Wavelength } \\ (\mathrm{nm})\end{array} & \begin{array}{c}\text { Oscillator } \\ \text { strength }\end{array} \\ 1 & 559.4 & 0.963800495 \\ 2 & 458.0 & 0.000000133 \\ 3 & 436.8 & 0.001465716 \\ 4 & 430.2 & 0.000000006 \\ 5 & 391.3 & 0.000000033 \\ 6 & 386.6 & 0.000000019 \\ 7 & 378.0 & 0.000030651 \\ 8 & 377.8 & 0.000140783 \\ 9 & 361.1 & 0.175086579 \\ 10 & 359.9 & 0.004932506 \\ 11 & 359.7 & 0.011581517 \\ 12 & 346.2 & 0.123776353 \\ 13 & 344.6 & 0.000005185 \\ 14 & 332.8 & 0.009964109 \\ 15 & 330.3 & 0.000000035 \\ 16 & 320.6 & 0.051382461\end{array}$

\section{DBOV-Ph}

$\begin{array}{ccc}\text { State } & \begin{array}{c}\text { Wavelength } \\ (\mathrm{nm})\end{array} & \begin{array}{c}\text { Oscillator } \\ \text { strength }\end{array} \\ 1 & 556.4 & 0.923050355\end{array}$




$\begin{array}{ccc}2 & 459.7 & 0.000000885 \\ 3 & 436.5 & 0.001304472 \\ 4 & 431.7 & 0.000004627 \\ 5 & 391.4 & 0.000001057 \\ 6 & 386.0 & 0.000001499 \\ 7 & 361.7 & 0.143953147 \\ 8 & 348.7 & 0.180054358 \\ 9 & 344.3 & 0.000018569 \\ 10 & 338.2 & 0.014303278 \\ 11 & 330.9 & 0.000056826 \\ 12 & 325.7 & 0.000003244 \\ 13 & 324.7 & 0.011439129 \\ 14 & 323.3 & 0.000219556 \\ 15 & 322.3 & 0.000000392 \\ 16 & 322.0 & 0.030813032\end{array}$

Line narrowing for the absorption: $500 \mathrm{~cm}^{-1}$.

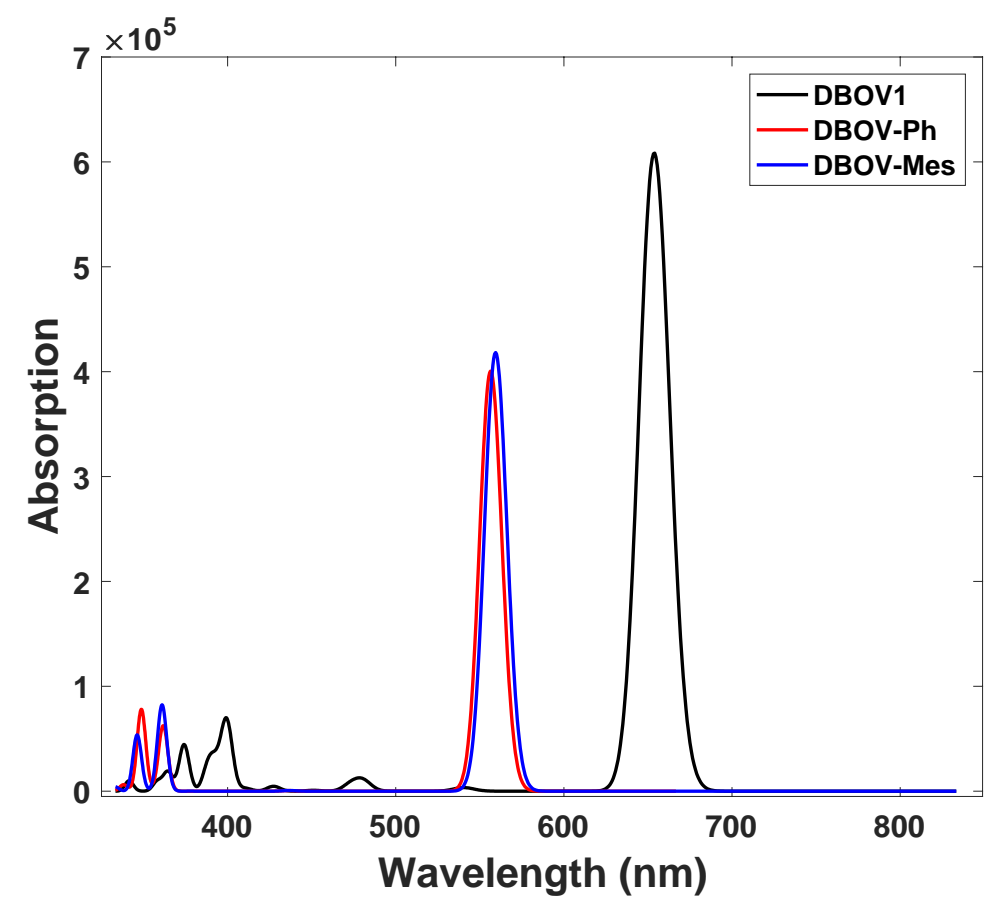

Figure 4. Absorption spectra of the DBOV molecules.

DBOV-Mes-C12 shows a red shift with respect to DBOV-Mes and DBOV-Ph. This trend agrees with the experimental absorption spectra.

XYZ coordinates of the optimized geometries

Coordinates for DBOV-Mes-C12

(164 atoms)
C -0.32426014148640
$-0.11820494221991$
$-0.13063678778650$
C -0.92693584605201
1.17936968329064
$-0.14705655307724$ 

C -0.42435197745742
2.23501748766912
0.67042164963996
C -1.05831594672751
3.52677797696903
0.62953953739271
C -0.26673945339317
4.66048950206671
0.97608010060467
C -2.42738718668177
3.59454574722689
0.10443701331813
C -2.61646989391585
2.75447384566245
$-1.04161295919871$
C -1.91994338374919
1.49544890265871
$-1.10323306471384$
C -2.24208530227915
0.54284293058749
C -1.69801202720425
$-0.77974944447132$
$-2.11662026707481$
$-2.05606850897786$
$-1.75000001057309$
C -1.59703777494518
$-3.12575524762530$
$-3.47842823530549$
C -0.65396494157280
3.12199534478032
C -3.39990571910404
$-4.84603952719199$
C -0.16207181482601
$-2.45242031130967$
C -0.20553551576296
$-5.10698201954179$
$-3.02050804094870$
$-2.88504923491601$
$-1.85813246973215$
$-2.18646983614917$
$-1.72602008369649$
$-0.96427503914555$
C 0.83498606150775
$-2.73892423833430$
$-0.71688892488024$
C 0.77996570279539
C 0.73910715279118
$-0.38162321776585$
C 0.76804338967775
2.03104369297913
C 0.94420725265105
4.44206061552583
C 1.30868761730921
$-4.07166733349990$
C 1.27231880300659
$-1.70261432136576$
C 1.28331338367146
0.66926462734042
C 1.41572884831962
3.16184001955947
C 2.34918486029556
$-4.30836418251222$
C 2.31172085828188
$-1.97913698840352$
C 2.31545274198374
0.35640083907924
C -2.04325305218514
$-4.12834225176886$
$-3.31535370843827$
0.01241687575354
0.79220759103486
1.43163578054642
1.64040737373772
0.11367557717852
0.87420033403481
1.58662006625319
1.94407767655535
1.09216398094790
1.81024001477775
2.49507751087872
$-3.74477982999530$
1.89278533885804
C 2.82590908793902
$-0.93517468763629$
C 2.80716705521528
$-5.43972021870737$
2.61376314508569
$-3.60611987836378$
$-5.84574712929245$
C -0.69162822330884
C -2.92423289571647
$-1.34224128233557$
C -3.41118858893501
$-0.04698335960668$
0.91365106196070
$-2.63032349565479$
$-4.07868393366129$
$-4.17032268219709$
$-3.18649430564707$
2.25744529261071
C -3.60678382901522
C -0.75084728566642
$-1.11814028674336$
3.12366547227195
H -6.53915039213303
H -2.03776086007194
$-19.01999124815825$
H -3.79478036538842
4.13831806861908
2.58187775946749
H -4.16936736813461
5.30624588741231
H 1.55504625426313
3.06277304688715
H 2.34975247874274
H 2.76943401805687
$-5.31074855842374$
H 2.73111052185914
1.13789554500242
H $\quad-2.76201820998140$
H 3.62168035051099
H 3.60045840840493
$-3.90733867307153$
$-3.52561194827315$
$-1.15309247683998$
$-3.22636108898343$
$-1.03669379718262$
0.11589741550652
$-5.38147085023546$
$-2.23709580837452$
$-4.10774295609736$
1.92245298434452
2.50040930388355
1.17746649717414
3.13401845060340
$-4.53448896404783$
2.61488412523675
3.33601423149642
$-4.29642969728122$
$-2.75728967676272$
$-4.85984223971401$
$-5.01188600093419$
H $\quad-4.05187658392768$
$-6.39721849564852$
$-2.05080891608856$
0.23505537122227
C 1.45919926327407
$-6.45466515363301$
$-0.48145697546577$
C 0.97502355728081
$-7.28845802197696$
0.55144140141453
C 2.60454704287087
$-6.84117073548907$
$-1.21598319972105$
0.79034889976495
C 1.60836940206795
$-8.51654841374453$
$-8.07303702729594$
$-0.93818183539812$ 

C 2.72321158481046
$-8.93453771545959$
0.05320115314664
C -0.17139408563644
$-6.85568205053632$
1.43903488427895
C 3.36661069988046
$-10.27705462706496$
0.31210488455525
C 3.16997894582188
$-5.94618420494234$
$-2.29486923017499$
H 1.22207986552206
$-9.16162457831324$
H 4.09507196794275
$-8.36761230455522$
H 0.17036529979839
$-6.12313550608861$
H $\quad-0.60094392586202$
$-7.71632425613902$
1.58787656543969
$-1.51470767092822$
2.19494121608073
1.97940047989923
H -0.97904504473942
$-6.36430219921689$
$-10.30861140680346$
H 4.40440355450390
H 2.81130788101945
$-11.09060021869182$
H 3.38353144644058
$-10.51787231473801$
H 3.45586274396059
$-4.95800634698180$
H 2.43061239523784
$-5.75655061993769$
$-7.34680597789946$
C -0.42990572541527
$-8.15860106570169$
$-9.67920937921466$
C -1.29169765879385
$-10.33497915444925$
0.87228710539509
$-0.06143139984983$
$-0.19272817822276$
1.38985842416741
$-1.89228351663894$
$-3.09384444498875$
$-2.63751064136451$
$-1.89376065741761$
$-1.92251503467835$
C -1.44193840651663
$-11.85812033045711$
$-3.30173437435573$
C -1.26043716899847
$-12.51687783824083$
$-3.27718626477734$
C -1.42440696907027
$-14.02897184708746$
$-4.65340638928264$
C -1.15279424960472
C -2.15093097429897
$-14.89010780031618$
$-4.68923348689539$
$-3.90412963455915$
$-16.39560104272439$
$-4.01698867274134$
$-17.25799269291588$
$-3.22936411494658$
C -2.87560677988925
$-18.76939478044566$
C -2.59891528750696
$-19.41883952306643$
C -2.75986216152838
$-7.61399578740762$
H 0.54916977284568
$-7.66365451314849$
$-7.92207832802160$
$-7.82381276260284$
$-10.14899919565028$
$-3.26775343807571$
$-4.64649386247634$
$-2.23209300343982$
Н -0.41947592134642
$-3.69484543662877$
H -2.50515442492954
$-2.32439718894413$
H -1.5512085051318
$-9.90693284291802$
H -0.28753948795111
$-9.90207236905763$
H -0.71068120437284
H -2.44381062596362
$-10.09418536293640$
$-0.84311940115531$
$-1.22855020493048$
$-1.51425081529412$
$-4.01092423781214$
$-3.71116340139062$
$-12.29335161091913$
$-2.56004411698114$
$-2.87850781194751$
H $\quad-0.25368961494816$
$-12.09659006852448$
$-12.01984373483443$
$-5.36685319813862$
$-12.32171754085365$
$-14.22278391428519$
$-5.03152156315825$
$-4.31235258811310$
$-5.74504386999147$
H -1.15283617962303
$-14.36246461186264$
$-14.67595507066740$
H -3.17694988799740
$-14.60331855595782$
$-4.26534778017534$
$-2.83528511435926$
$-3.65795124780734$
H -0.85368354281255
$-16.60861531500683$
$-16.68293861678713$
$-5.08575335093302$
$\begin{array}{ll}\mathrm{H} & -1.89354342363805 \\ \mathrm{H} & -3.90158014026980\end{array}$
$-17.06720403827221$
$-3.60401981897318$
H -2.87375240634683
$-16.92473805059970$
H -3.28271757363920
$-19.26941288774409$
$-2.17337677570977$
$-2.55624551792005$
$-2.88887095374747$
$-18.96017170687386$
$-19.24869878085635$
H -3.77580675592293
H $\quad-2.60230262213148$
$-20.51144122404915$
C -3.48653611613091
4.42238884229958
$-5.05146840625021$
$-4.59141209121050$
0.69518901033070
$\begin{array}{ll}4.43026983668916 & 0.21121676252647\end{array}$
5.21060634651325
C -3.29047336655381
5.21060634651325
5.51232462582643
1.89649485448839
0.52421239002502
6.51206376105158
1.43349102621902
C -5.32470336160000
6.26219142127240
2.18040828173479 


\begin{tabular}{|c|c|c|c|}
\hline & -2.32202926166047 & & \\
\hline & -6.19741256386817 & & \\
\hline & -5.57324957735842 & & \\
\hline & -6.69415397056899 & & \\
\hline & -3.96754988089043 & & \\
\hline & -2.02469004155009 & & \\
\hline & & & \\
\hline & -1.39571542012590 & & \\
\hline & -7.25466183851688 & & \\
\hline & -5.87934305292282 & & \\
\hline & -6.14345619961288 & & \\
\hline & -5.01673692401218 & & \\
\hline & -5.80522445678296 & & \\
\hline$C$ & -0.594 & & \\
\hline & & & \\
\hline & & & \\
\hline & & & \\
\hline & & & \\
\hline & & & \\
\hline & & & \\
\hline & & & \\
\hline & & & \\
\hline & & & \\
\hline & & & \\
\hline & & & \\
\hline & & & \\
\hline & & & \\
\hline & & & \\
\hline & & & \\
\hline & & & \\
\hline & & & \\
\hline & & & \\
\hline & & & \\
\hline & & & \\
\hline & & & \\
\hline & & & \\
\hline $\mathrm{H}$ & & & \\
\hline & & & \\
\hline $\mathrm{H}$ & & & \\
\hline $\mathrm{H}$ & & & \\
\hline $\mathrm{H}$ & & & -7.8 \\
\hline $\mathrm{H}$ & & & -9.30 \\
\hline $\mathrm{H}$ & 3.99 & & -8.65 \\
\hline $\mathrm{H}$ & & & -9.60 \\
\hline $\mathrm{H}$ & & & -10.2 \\
\hline $\mathrm{H}$ & & & \\
\hline & & & \\
\hline$\Pi$ & & & -12.68217123481013 \\
\hline$\Pi$ & & & \\
\hline & 6.29036978376228 & 6.26117686408089 & -12.03024923071176 \\
\hline
\end{tabular}

Coordinates for DBOV-Mes (92 atoms) 

C -0.81752094824052
$-0.05179227598906$
0.59485136327361
C -1.51719179147868
1.20698130718531
0.51764462697142
C -0.82742661854085
2.43358511371633
0.75506481958920
C -1.54058680458701
3.68076474044698
0.66952160377467
C -0.83333549901470
4.89113791544584
0.90443258081003
C -2.93506879290497
3.69241507781421
0.35404705654683
C -3.60720362599070
2.48406159112560
0.12503777575526
C -2.89857092264014
1.22817328166549
0.20328795476754
C -3.59370980870379
$-0.00037499099721$
$-0.03798973862006$
C -2.90218357884393
$-1.25134458297880$
0.03668306575917
C -3.60373702595964
$-2.46866548102241$
$-0.20721245443724$
C -2.89049275971906
$-3.74803773114481$
$-3.75012229725647$
$-0.12583637872114$
C -1.49245443236795
2.45653482735887
$-4.99740293243022$
C -0.77958423597992
$-2.52348051677640$
C -0.80272190668127
$-5.00905840905158$
C 0.61463215682616
$-2.54467044987949$
C 0.57856355204516
$-0.06512917852712$
C 0.57085792109901
2.43159360984402
4.87233768285285
C 0.51291120269111
C 1.28690375454176
$-3.80063918798885$
C 1.27380501212735
$-1.31615168862314$
C 1.28390180705165
1.15215819108908
3.65620951821355
C 1.20842009559650
$-3.77313728753268$
C 2.69467816422161
C 2.66469257081069
$-1.33982046847795$
C 2.65598110192499
1.08399702955551
C -3.52775622419731
$-4.97256620943158$
0.19796416218558
$-0.19546980903123$
0.28469323750569
0.43525047069006
0.60130333532882
0.75009655099704
0.91584669861858
1.07782796408517
1.21626340720783
0.82949790017525
0.99108253764674
1.15975398023527
1.30409243923025
1.15032257169768
1.31047340554265
1.47536503383643
$-0.35334860758560$
$-2.60150598073075$
1.38095113077991
C 3.34794515070602
$-0.12534408485293$
1.54941741409107
C -2.83254778551380
$-6.18883371431313$
C -1.48686184069270
$-6.20776333902928$
$-2.40053976402560$
$-1.19119643283550$
0.02328992457440
$-0.26428960665373$
0.04974725535621
$-0.52177437579563$
$-0.59544211027333$
C $\quad-5.64951317985156$
C -4.98473498681925
1.28490661621337
C $\quad-5.66815959901417$
1.28490661621337
-1.26469473589191
$-0.35679293021842$
$-0.42660794086980$
C -1.50227207946706
$-6.31492292258610$
C 1.35024419833850
C 1.46794335351492
$-6.98029762375047$
C 1.92952020618004
C 2.16215198765040
$-6.88183560757891$
$-8.19870376868661$
C 2.61388839315711
$-8.09931995811984$
C 2.74341161805680
$-8.77680649513119$
C 0.86431664506173
$-6.39394563276270$
C 3.49018642106474
$-10.08863455657714$
0.35756604883163
0.68848575864716
1.92887058394536
$-0.47166512868269$
1.98756169714617
$-0.36702752029517$
0.85378110063134
3.18582054958589
0.93258099488598
$-6.18962274808016$
$-1.81168432255577$
C -4.13451633930909
4.86878426005775
2.77029162220430
8.76108887842877
0.02267816224157
5.08645311596386
$-2.22614964614647$
C -3.18002030615284
6.88813284064076
$-1.02395024888059$
7.46314029426425
0.11231507545051
6.78308565223295
5.66902715629712
1.32997786791928
C -4.93062302383412
5.56232770127361
$-0.96906479452836$
1.43100718904133
C -4.24843158590799
4.99812992640233
0.27027165858650 


$\begin{array}{clcc}\text { H } & -1.36906277763655 & 5.84023868205269 & 0.83557894193056 \\ \text { H } & -5.55187271563222 & 3.40568732575160 & -0.25065240029585 \\ \text { H } & 1.04540015607428 & 5.81090748555314 & 1.39793293311863 \\ \text { H } & 2.26879005774388 & 3.68702875510327 & 1.55642931131295 \\ \text { H } & 3.23143699340508 & -4.72229353698204 & 1.20619581373728 \\ \text { H } & 3.21711654372018 & 1.99821255449619 & 1.67084539142203 \\ \text { H } & -4.58765065444022 & -5.00338136960537 & -0.60764179951202 \\ \text { H } & 4.41437999063441 & -2.60126277555175 & 1.62718355947302 \\ \text { H } & 4.39481484047628 & -0.14129163882943 & 1.79894187386428 \\ \text { H } & -3.36514801876752 & -7.12719421859546 & -0.44670363519426 \\ \text { H } & -0.95109807712101 & -7.15671531741802 & 0.12040531399241 \\ \text { H } & -5.53739502567962 & -3.31482465971618 & -0.71631813779770 \\ \text { H } & -6.71513263793828 & -1.17530496371019 & -0.84417338699538 \\ \text { H } & -6.73465062433072 & 1.28456776017741 & -0.67261417951051 \\ \text { H } & 2.24870901453213 & -8.71021590760297 & 2.95234982695412 \\ \text { H } & 3.06077263627479 & -8.53124709101291 & -1.26937477023739 \\ \text { H } & 1.31685609887335 & -5.41687693738789 & 3.43355287249282 \\ \text { H } & 1.01511464595742 & -7.06476061270916 & 4.04849799009815 \\ \text { H } & -0.22090849914453 & -6.22030281278646 & 3.07378043955432 \\ \text { H } & 3.48608013759472 & -10.49621903129596 & 1.95777489504072 \\ \text { H } & 4.54520188873759 & -9.97129898598121 & 0.62162628926883 \\ \text { H } & 3.04166356761001 & -10.84823418769956 & 0.26564937985783 \\ \text { H } & 2.34323099089520 & -6.75739484657182 & -2.59680423357074 \\ \text { H } & 2.24616651091025 & -5.17259153755888 & -1.78100301823854 \\ \text { H } & 0.76206908193375 & -6.07677704085321 & -2.12172598653993 \\ \text { H } & -4.65877886865646 & 5.43694499012453 & 3.55684778006918 \\ \text { H } & -3.08004716346639 & 4.75295394424475 & 3.07873083228973 \\ \text { H } & -4.56706598961715 & 3.85296853695613 & 2.73920855356588 \\ \text { H } & -5.44751969575356 & 9.40344215224739 & -0.78990279355804 \\ \text { H } & -5.77298101974690 & 9.33137046324823 & 0.96644931400622 \\ \text { H } & -6.90236617310147 & 8.57767437048503 & -0.18324201697200 \\ \text { H } & -3.33441735510589 & 5.75638126250168 & -3.08885531430563 \\ \text { H } & -3.62714006247958 & 4.10690193717760 & -2.47371433983311 \\ \text { H } & -2.09383865525395 & 4.91843918806940 & -2.11408406603115 \\ \text { H } & -4.55853041068043 & 7.40436761128233 & -1.98697381780243 \\ \text { H } & -5.37272164844846 & 7.21514525279426 & 2.23437954606591\end{array}$

Coordinates for DBOV-Ph

(74 atoms)
C -4.44003008021391
$-3.77072354297747$
$-0.04020273486989$
C -3.04823707067101
$-3.95110096766737$
C -2.16997146654773
$-2.86255355411663$
C -2.71799238581052
$-1.53678442916186$
C -4.14568361286671
$-1.35322659655404$
C -4.98401356430087
$-2.50074528240234$
C -1.85785312477516
$-0.39786326110412$
0.90411672687686
C -2.41619619547711
1.08106675621172
C -3.84982336760935
$-0.03501163486066$
C -4.69693087994051
2.04895496408543
C -1.55599443587028
3.35948464671945
C -2.12031617775775
3.50888467092160
C -3.54860942582782
2.42672464883555
$-0.02902593190755$
$-0.00890296645920$
$-0.00100445078462$
$-0.00351923092868$
$-0.02709239334093$
0.00574397081715
0.01274802227595
0.01932910174915
0.01104407075073
0.01552151050521
0.02744980972394
0.04374026040503
0.04143440237863 

C -0.71327490253873
$-3.03398618488902$
0.00376527264667
C 0.13380640255528
$-1.88705370447122$
0.00726677830270
C -0.42498993089193
$-0.56434271669092$
C 0.42498891342064
0.56433048271087
0.00496867010853
C -0.13380703077836
1.88704178130288
0.00498996375145
C 0.71327430788996
3.03397441772602
0.00734505207916
C 0.11499743748276
4.30999617259540
0.00388642869096
C -1.26148812382338
4.47109853398769
0.01457352641066
C -0.11499805500319
$-4.31000812381387$
0.02716487878097
0.01439533713462
C 1.26148768698284
C 2.12031561592813
$-4.47111103266352$
0.02697082548352
$-3.35949760766599$
0.02729733668618
C 1.55599373513391
$-2.04896719524899$
0.01542760986052
C 2.41619544075481
$-0.90412899821249$
0.01269712169673
C 1.85785220051360
0.39785141569385
0.00574938060586
C 2.71799170874876
1.53677312079692
2.86254274108593
$-0.00095720436263$
C 2.16997094676599
C 3.54860907575873
$-3.50889823120324$
$-0.00879835430715$
0.04357023963906
$-2.42673853238699$
0.04130479699597
C 3.84982284706298
$-1.08107925199022$
0.03499965637638
0.01926229749340
C 4.69693053382057
C 4.14568302478483
1.35321584616551
0.01101850912269
2.50073548554944
$-0.00348696877409$
C 4.98401332501724
C 4.44002962940793
C 3.04823665762093
3.77071421551499
3.95109093300549
$-0.02701696133859$
$-0.04007225604468$
C -6.18467089244481
0.14404717832115
$-0.02888160668690$
C 6.18467164461696
$-0.14405886585859$
0.01758585294508
C -6.90917677823144
0.09255680300229
$-0.36643370536407$
0.01754636738725
C 6.88792833026853
C 8.27627359548334
$-0.53564316603408$
1.22121494347772
$-1.17926653929003$
C 8.98518582662037
$-0.48482916557637$
$-0.26215493498087$
$-1.17325392488060$
0.03131551403574
1.22880098992504
$\begin{array}{ll}\text { C } & 8.29739137817826 \\ \text { C } & 6.90917654899285\end{array}$
$-0.09266468084896$
0.36652132990323
1.22118059104610
C -6.88792589413380
0.53573252873670
$-1.17920956767924$
C $\quad-8.27627073631491$
C -8.98518472891723
0.48482224951787
$-1.17318504635107$
0.03137987591322
0.26204991605490
C -8.29739141843082
H $\quad-5.09527008214191$
$-4.64677734129433$
$-4.97052096849232$
H -2.66182653587747
H -6.06744370241260
$-2.36844445398643$
4.52122905286947
2.56460476194572
H $\quad-5.45607846761153$
H $\quad 0.73740663765408$
5.20574887847450
H -1.69251417342102
5.47701620113604
$-5.20576112430426$
$\begin{array}{ll}\mathrm{H} & -0.73740677248571 \\ \mathrm{H} & 1.69251375850357\end{array}$
H 3.96479918894029
H 5.45607886900993
H 6.06744373415054
H 5.09526893998324
H 2.66182615859217
$-5.47702907164221$
1.22884779731603
$-0.06029178692699$
$-0.03996242007548$
$-0.03728598948616$
0.06036488239578
0.05751859675015
0.01551139753316
0.03761344560208
0.01529930598037
$-4.52124336761837$
0.03737325956269
$-2.56461859062445$
0.06014593715524
0.05737387711275
2.36843491953516
4.64676911908773
$-0.03722012768734$
$-0.06012731084139$
$4.97051149714185 \quad-0.03977528670204$
H $\quad-6.37510199721684$
$-0.08038625105032$
2.16066495390385
$-0.40814025790278$
$-2.12378757788377$
H $\quad 6.33659412032837$
H 8.80571050854937
$-0.70991886409442$
$-2.11527607097133$
H 10.07139749405708
H $\quad 8.84387208862548$
$-0.62046785567283$
0.03725168757596
$-0.22152553338962$
2.17647774543775
0.08020011654944
2.16064419986707 


$\begin{array}{clcc}\mathrm{H} & -6.33659014013157 & 0.40830375947426 & -2.12372613070022 \\ \mathrm{H} & -8.80570684020018 & 0.71008530610597 & -2.11519351118387 \\ \mathrm{H} & -10.07139632530213 & 0.62046198611918 & 0.03732501269608 \\ \mathrm{H} & -8.84387298345144 & 0.22134622667880 & 2.17652094274992\end{array}$

\section{Reflectance spectra for the DBOV:PS 1 wt.\% films}

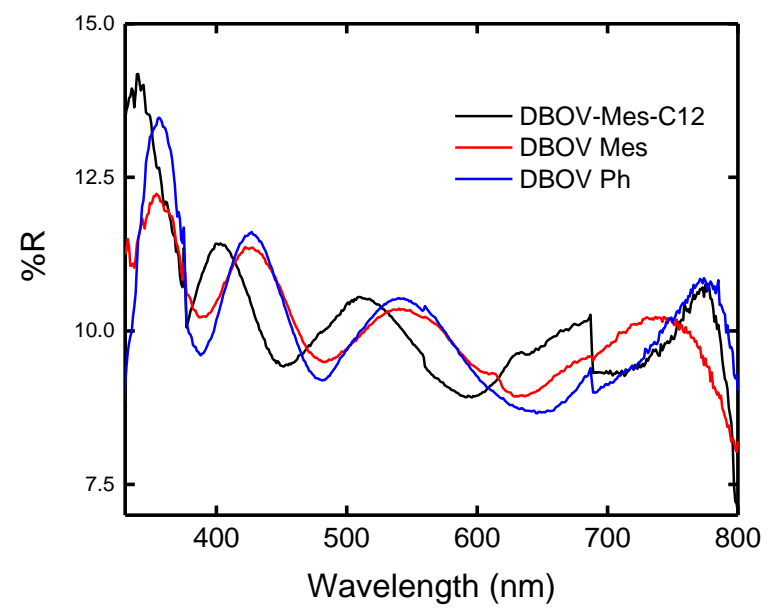

Figure S4. Reflectance spectra of the DBOV: PS films (1 wt.\% ratio).

\section{TA spectra on $0.01 \mathrm{mg} / \mathrm{mL}$ solutions}

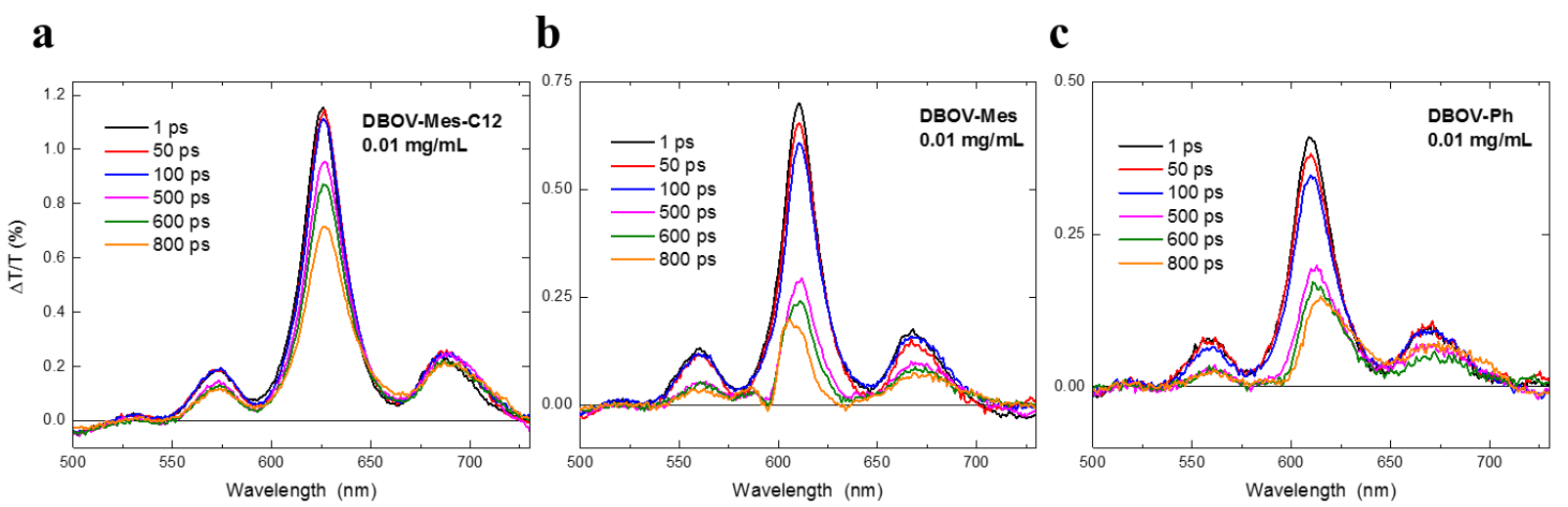

Figure S4. TA spectra as a function of pump-probe delay for DBOV-Mes-C12 (a) DBOV-Mes (b) and DBOV-Ph (c) $0.01 \mathrm{mg} / \mathrm{mL}$ in toluene. Note the clear difference in the photodynamics among the derivatives, with DBOV-MES-C12 exhibiting the slowest dynamics even at such low concentration. 


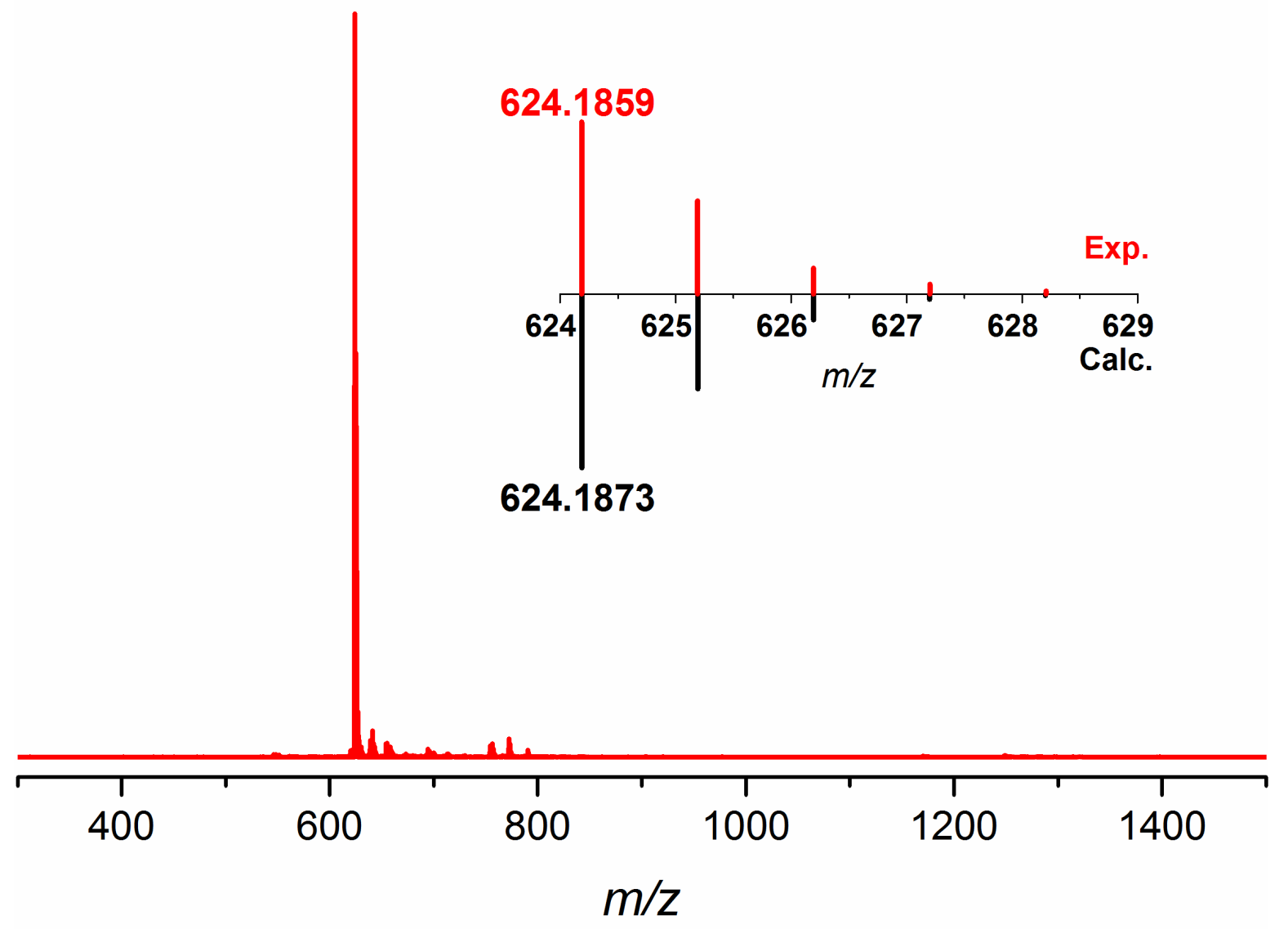

Figure S5. High-resolution mass (MALDI) spectra of DBOV-Ph with 7,7,8,8tetracyanoquinodimethane (TCNQ) as matrix. The inset shows good agreement between isotopic distribution patterns of experiment and calculation.
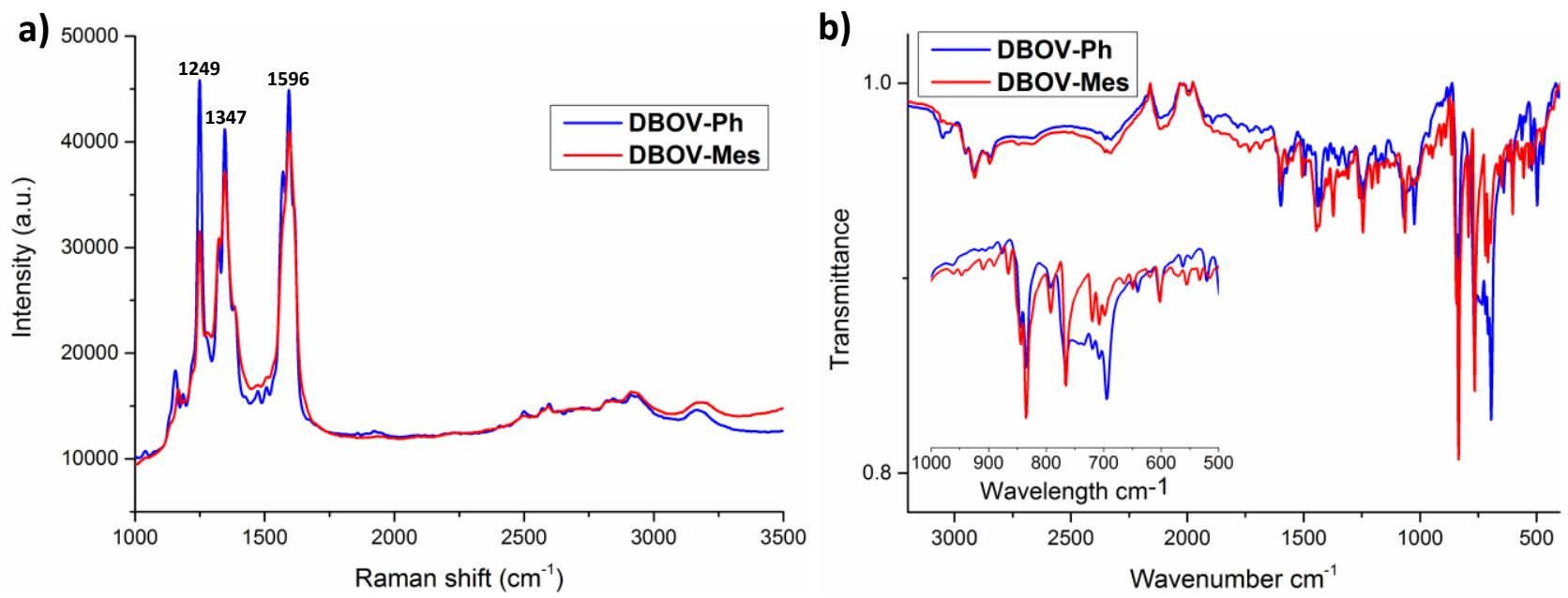

Figure S6. (a) Raman and (b) FT-IR spectra of DBOV-Ph in comparison with DBOVMes(inset shows magnified spectra from 500 to $1000 \mathrm{~cm}^{-1}$ ). The good agreement of the spectral features further corroborates the chemical identity of DBOV-Ph, in comparison to DBOV-Mes, which could be unambiguously characterized by NMR. ${ }^{1}$ 


\section{References}

1. $\quad$ Coles, D. M.; Chen, Q.; Flatten, L. C.; Smith, J. M.; Mullen, K.; Narita, A.; Lidzey, D. G. Nano Lett 2017, 17, (9), 5521-5525.

2. $\quad$ Paterno, G. M.; Chen, Q.; Wang, X. Y.; Liu, J.; Motti, S. G.; Petrozza, A.; Feng, X.; Lanzani, G.; Mullen, K.; Narita, A.; Scotognella, F. Angew Chem Int Ed Engl 2017, 56, (24), 6753-6757.

3. Hanwell, M. D.; Curtis, D. E.; Lonie, D. C.; Vandermeersch, T.; Zurek, E.; Hutchison, G. R. J Cheminform 2012, 4, (1), 17.

4. $\quad$ Neese, F. Wires Comput Mol Sci 2012, 2, (1), 73-78.

5. $\quad$ Lee, C. T.; Yang, W. T.; Parr, R. G. Phys Rev B 1988, 37, (2), 785-789.

6. $\quad$ Schafer, A.; Horn, H.; Ahlrichs, R. J Chem Phys 1992, 97, (4), 2571-2577.

7. Schafer, A.; Huber, C.; Ahlrichs, R. J Chem Phys 1994, 100, (8), 5829-5835.

8. $\quad$ Eichkorn, K.; Weigend, F.; Treutler, O.; Ahlrichs, R. Theoretical Chemistry Accounts 1997, 97, (14), 119-124.

9. $\quad$ Pavosevic, F.; Neese, F.; Valeev, E. F. J Chem Phys 2014, 141, (5), 054106. 